\title{
Transcriptome analyses of adult mouse brain reveal enrichment of IncRNAs in specific brain regions and neuronal populations
}

\author{
Beena M. Kadakkuzha ${ }^{1}$, Xin-An Liu ${ }^{1}$, Jennifer McCrate ${ }^{1}$, Gautam Shankar ${ }^{2}$, Valerio Rizzo ${ }^{1}$, \\ Alina Afinogenova ${ }^{1}$, Brandon Young ${ }^{3}$, Mohammad Fallahi' ${ }^{2}$, Anthony C. Carvalloza ${ }^{2}$, \\ Bindu Raveendra ${ }^{1}$ and Sathyanarayanan V. Puthanveettil ${ }^{1 *}$
}

${ }^{1}$ Department of Neuroscience, The Scripps Research Institute, Jupiter, FL, USA

2 Informatics Core, The Scripps Research Institute, Jupiter, FL, USA

${ }^{3}$ Genomics Core, The Scripps Research Institute, Jupiter, FL, USA

Edited by:

Francesco Moccia, University of

Pavia, Italy

Reviewed by:

Hermona Soreq, The Hebrew

University of Jerusalem, Israel

Beena Pillai, CSIR-Institute of

Genomics and Integrative Biology,

India

${ }^{*}$ Correspondence:

Sathyanarayanan V. Puthanveettil,

Department of Neuroscience, The

Scripps Research Institute, 130

Scripps Way, Jupiter, FL 33458, USA

e-mail: sputhanv@scripps.edu
Despite the importance of the long non-coding RNAs (IncRNAs) in regulating biological functions, the expression profiles of IncRNAs in the sub-regions of the mammalian brain and neuronal populations remain largely uncharacterized. By analyzing RNASeq datasets, we demonstrate region specific enrichment of populations of IncRNAs and mRNAs in the mouse hippocampus and pre-frontal cortex (PFC), the two major regions of the brain involved in memory storage and neuropsychiatric disorders. We identified 2759 IncRNAs and 17,859 mRNAs in the hippocampus and 2561 IncRNAs and 17,464 mRNAs expressed in the PFC. The IncRNAs identified correspond to $\sim 14 \%$ of the transcriptome of the hippocampus and PFC and $\sim 70 \%$ of the IncRNAs annotated in the mouse genome (NCBIM37) and are localized along the chromosomes as varying numbers of clusters. Importantly, we also found that a few of the tested IncRNA-mRNA pairs that share a genomic locus display specific co-expression in a region-specific manner. Furthermore, we find that sub-regions of the brain and specific neuronal populations have characteristic IncRNA expression signatures. These results reveal an unexpected complexity of the IncRNA expression in the mouse brain.

Keywords: IncRNA, mRNA, genomic context, hippocampus, pre-frontal cortex, differential gene expression, RNAseq, pathway analysis

\section{INTRODUCTION}

While previously regarded as simply "transcriptional noise," noncoding RNAs (ncRNAs) constitute $97-98 \%$ of the human genome that is not translated into proteins (Derrien et al., 2012). Long non-coding RNAs (lncRNAs) are transcribed by RNA polymerases II and III (Dieci et al., 2007; Kapranov et al., 2007; Guttman et al., 2009), and are a minimum of 200 nucleotides long. Closer scrutiny of lncRNAs, spurred by recent technological advances in DNA microarrays and RNA sequencing, has suggested that they are involved in chromatin remodeling, transcription, alternative splicing, imprinting, cytoplasmic trafficking, and aging (Mercer et al., 2009; Wang and Chang, 2011; Soreq et al., 2014). Based on the genomic orientation of lncRNAs and their associated protein-coding genes, IncRNAs are characterized as intronic, bidirectional, antisense, sense overlapping, and intergenic (Ma et al., 2013).

Whole transcriptome sequencing to investigate tissue-specific expression in lower organisms found that the largest number

Abbreviations: DE, Differential expression; FPKM, fragments per kilobase of transcript per million fragments sequenced; GEO, Gene Expression Omnibus; LCM, Laser capture microdissection; LncRNA, Long non-coding RNA; LincRNA, long intergenic non-coding RNA; ncRNA, Non-coding RNA; PFC, Pre-frontal cortex; qRT-PCR, Quantitative real-time polymerase chain reaction. of tissue specific lncRNA transcripts is expressed in the brain (Kaushik et al., 2013). The large number of lncRNAs that exhibit neuronal-specific expression suggests their essential role in the neuronal diversification seen in higher vertebrates (Cao et al., 2006; Qureshi et al., 2010). It has been reported that aberrant expression of lncRNAs is associated with neurological disorders (Faghihi et al., 2008; Khalil et al., 2009; Johnson, 2012; Pauli et al., 2012; Roberts et al., 2014; Soreq et al., 2014). Using the in situ hybridization data available from the Allen Brain Atlas, Mercer et al., reported that lncRNAs are differentially expressed in different regions of the adult mouse brain (Mercer et al., 2008). Recently, Sauvageau et al., have developed several lines of knockout mice to investigate a family of non-coding RNA molecules known as long intergenic non-coding RNAs (lincRNAs) (Sauvageau et al., 2013). These studies reveal that lincRNAs regulate key functions of the overall viability and developmental processes of mice, including the development of the cerebral cortex.

While functional characterization of neuronal-enriched lncRNAs is still limited, broader studies of lncRNA function have implicated them as regulators of transcription through epigenetic regulation of chromatin structure and RNA-transcription factor interaction (Kurokawa, 2011; Kornienko et al., 2013; Wu 
et al., 2013; Halley et al., 2014). Despite this importance we still do not have unbiased estimates of lncRNAs expressed in specific brain regions and the extent of their differential expression in mammalian brain.

Here we report the identification of the lncRNAs and mRNAs expressed in two key brain regions involved in memory and neuropsychiatric disorders, the hippocampus and pre-frontal cortex (PFC). Following RNAseq analysis $(N=4$ biological replicates) of hippocampus and PFC, we identified 2759 lncRNAs expressed in the hippocampus and 2561 lncRNAs in PFC using a customized computational analysis pipeline and have identified their chromosomal locations. Among these, 2390 lncRNAs are expressed in both regions. Importantly, 24 lncRNA transcripts are differentially expressed in the hippocampus and PFC. We have then studied differential expression of a subset of lncRNAs in (1) two other brain regions, striatum and cerebellum, (2) dorsal and ventral hippocampus, (3) three different neuronal populations (CA1, CA3, and dentate gyrus neurons of hippocampus), and (4) have examined subcellular distribution of a subset of candidates in the hippocampus and PFC by RNA in situ hybridization and sub-cellular fractionation. Our studies establish that specific brain regions and neuronal populations have characteristic expression pattern of lncRNAs.

\section{RESULTS}

\section{OVERVIEW OF InCRNA AND mRNA PROFILES IN ADULT MOUSE HIPPOCAMPUS AND PFC}

To perform an unbiased profiling of the total transcriptome of the hippocampus and pre-frontal cortex (PFC) in the adult mouse, RNA samples were prepared from the respective brain regions of $7-9$ week old male mice ( $N=4$ biological replicates, each replication has two mice). The transcripts were assembled and analyzed using a reported workflow as shown in Figure 1A. We mapped a total of 20,618 genes in the hippocampus and 20,025 genes in the PFC, including both protein-coding and non-coding transcripts (Table S1). There are $3823 \operatorname{lncRNAs}$ so far annotated in the mouse genome, according to the NCBIM37, and the 2759 lncRNAs mapped in the hippocampus and 2561 in the PFC from our study represent $\sim 70 \%$ of the total annotated mouse lncRNAs. Alignment of mapped reads from each sample to mouse transcriptome is provided in Table S1. Additionally, these lncRNAs represent $\sim 14 \%$ of the total mouse brain transcriptome mapped in this study. The total transcriptome composition in each tissue is shown in Figures 1B,C for the hippocampus and PFC, respectively. Next, we estimated the expression abundance of all lncRNA and mRNA genes by running Cufflinks across 8 samples using the ENSEMBL annotation file (NCBI37).

Analysis of the top 50 lncRNAs based on expression abundance identified key members such as MALAT1, MEG3, RIAN, XIST, MIAT, and TUG1 (Figures S1A,B). MALAT1 knockout mice studies showed that MALAT1 neighboring genes were deregulated, indicating a potential cis-regulatory role of MALAT1 in gene transcription (Zhang et al., 2012). The Allen Brain Atlas shows a similar expression profile for the lncRNAs MEG3, RIAN, and MIAT. Additionally, we looked at the top 50 mRNAs and the complete list is represented in Figures S1C,D. This abundance estimation indicated much lower expression levels of non-coding
RNAs compared to that of protein-coding RNAs and that most lncRNAs $(>80 \%)$ are expressed at a low level, an observation similar to those drawn from previous studies of the mammalian genome (Mercer et al., 2008; Derrien et al., 2012).

The transcriptomic profile of mRNA and lncRNA expression is summarized in Table S1. Further classification of the lncRNAs based on genomic context using the ENSEMBL annotation file provided eight biotypes: antisense, long lincRNAs, processed transcripts, sense overlapping, sense intronic, long non-coding, $3^{\prime}$ overlapping ncRNA and ncRNA host. Among these, lincRNAs (53\%) and antisense ncRNAs (38\%) showed predominance compared to other types of ncRNAs in both tissues (Figures 1D,E), an outcome similar to the observations from earlier studies (Khalil et al., 2009; Pauli et al., 2012; Ma et al., 2013). For example, the human GENCODE catalog mapped more than $70 \%$ of the lncRNAs into the lincRNA category (Derrien et al., 2012). Similarly the transcriptomic profiling in Maize showed that $93 \%$ of $\operatorname{lncRNAs}$ are located in intergenic regions and only $7 \%$ of the lncRNAs overlap with gene sequences (Li et al., 2014). To estimate the differential expression (DE), the raw read counts generated in HTSeq were used in the R Bioconductor package DESeq. DESeq analyses of the protein coding as well as non-coding transcripts showed a robust DE pattern where 24 long non-coding (Figure 2A) and 821 protein-coding transcripts were differentially expressed between the hippocampus and PFC (adjusted $p$-value $<0.05$; Table S1).

\section{CHROMOSOME ORGANIZATION OF InCRNA GENES IN MOUSE BRAIN}

Next, we asked whether the differentially enriched lncRNAs in the hippocampus and PFC are clustered in a specific region or randomly distributed on chromosomes. In order to observe the chromosomal distribution of lncRNAs, we mapped the transcriptional loci of all 2930 lncRNAs detected in this study. Table S2 list the number of lncRNAs expressed from each chromosome from the current study and Figure S3 shows the distribution of lncRNAs on each chromosome. Based on the number of lncRNAs encoded, the top three chromosomes (2, 4, and 11) are highlighted in red. A "spot" on the chromosome where we observe a large number of lncRNAs are clustered together, we termed as an "IncRNA transcriptional cluster" while discussing the chromosomal distribution of lncRNAs in the manuscript. Figure 3 shows that most of the lncRNA loci mapped in this study are clustered along chromosomes 2, 4, and 11. LncRNA loci in other chromosomes are shown in Figure S3. We find that though lncRNA loci are present as several clusters, the genomic loci of differentially expressed lncRNAs in the hippocampus and PFC are randomly distributed along the chromosomes (red: higher in PFC and blue: higher in hippocampus). Interestingly, we did not find any hippocampal or PFC lncRNAs present in the Y chromosome. Similar to our finding, an earlier study on the mouse embryonic brain also did not assign any lncRNAs to the Y chromosome (Lv et al., 2013).

\section{EXPRESSION ANALYSIS OF DIFFERENTIALLY ENRICHED PROTEIN-CODING-NONCODING GENES THAT SHARE COMMON GENOMIC LOCUS}

We analyzed whether the differentially enriched lncRNAs and the mRNAs within its vicinity show co-enrichment in these 


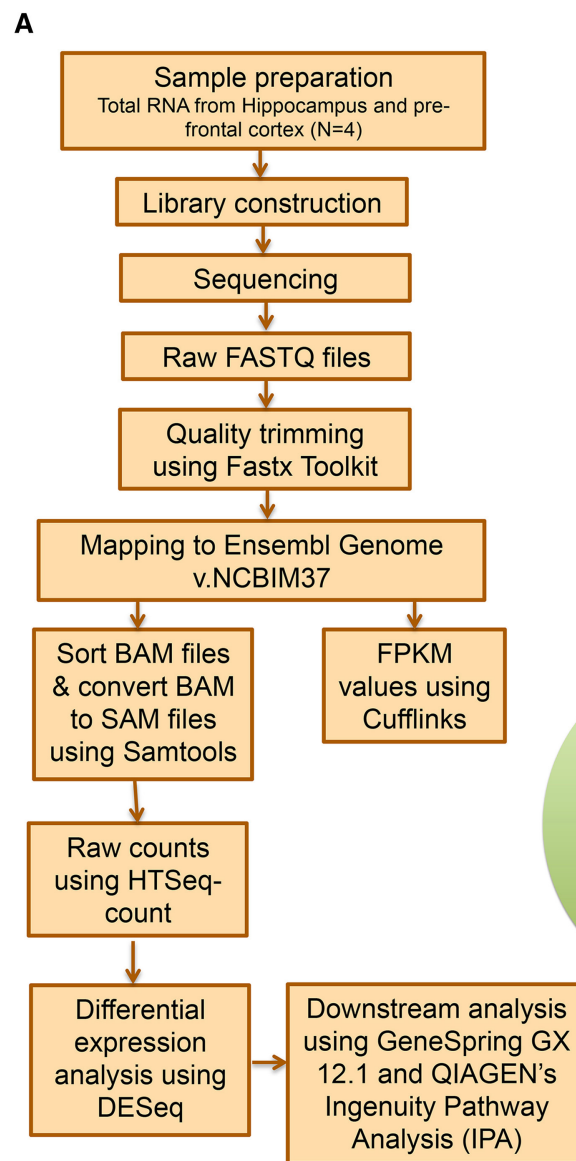

FIGURE 1 | Next generation sequencing analysis of hippocampus (HP) and pre-frontal cortex (PFC) from adult mouse brain. (A) Pipeline for the identification of mRNAs and IncRNAs from mouse brain total RNA preparation. Total RNA was isolated from HP and PFC for RNAseq $(N=4)$.

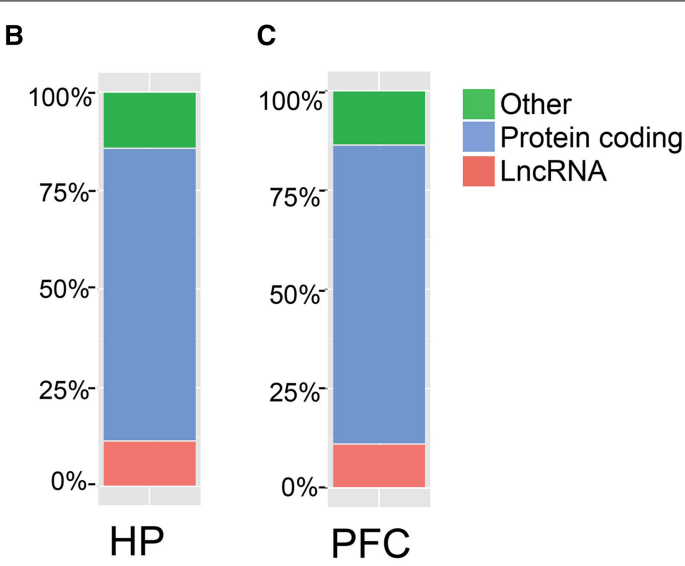

D

E

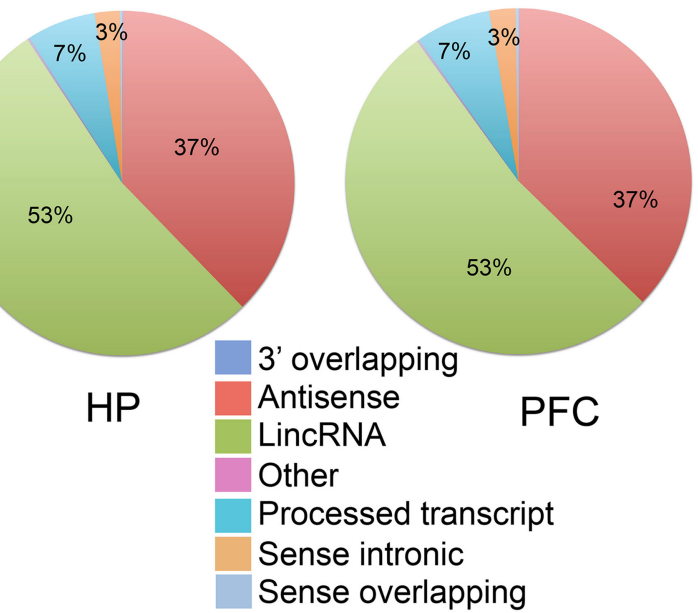

brain regions. Selective enrichment of lncRNA and neighboring mRNAs suggest co-regulation of their expression. We determined which of the 24 differentially expressed lncRNAs are transcribed from genomic loci overlapping with, or adjacent to, the loci that encode 821 differentially expressed mRNAs identified from DESeq analysis. For the purpose of defining "genomic association," we have defined $50 \mathrm{~Kb}$ in either direction of the genomic loci of a given lncRNA (Figure 2B) as the "neighboring chromosomal region." Furthermore, we considered that the DE of one of the genes in this pair (lncRNA and mRNA) is either positively or inversely correlated with the DE of the other gene.

By screening $\sim 50 \mathrm{~Kb}$ genomic region flanking the genomic loci of all 24 lncRNAs on either direction using the UCSC genome database, (https://genome.ucsc.edu/), we identified $\sim 50 \mathrm{mRNA}$ genes. Matching these $\sim 50 \mathrm{mRNAs}$ to the differentially enriched 821 mRNA gene list identified 9 mRNAs, that are within $50 \mathrm{~kb}$ of lncRNAs and are differentially enriched between the hippocampus and PFC. For further characterization we have selected from this list, lncRNAs and their corresponding mRNAs coded
(B, C) Classification of whole transcriptome from HP and PFC, respectively, based on the coding potential of the transcripts mapped. (D, E) Pie charts showing the percentage of seven categories of IncRNAs identified in HP and PFC on the basis of their relationship with protein coding genes. from the same loci. We found three lncRNA-mRNA pairs that share a common genomic locus and are partially or completely overlapping with each other.

One of these three pairs, GM9968, is an lncRNA significantly enriched in the mouse hippocampus and transcribed in the antisense direction of DNA binding protein gene ZBTB20 (Zinc finger and BTB domain-containing protein 20). Past studies showed that ZBTB20 is particularly expressed in primary hippocampal neurons, cerebellar granule cells, gliogenic progenitors and differentiated glia. RNAseq analyses showed that both of these transcripts are enriched in the hippocampus, and we confirmed the results by QRT-PCR, which showed a 2 -fold enrichment of GM9968 and 3-fold enrichment of ZBTB20 in the hippocampus compared to the PFC (Figure 2C). In a similar way we analyzed two more protein-coding lncRNA pairs to identify specific expression patterns. GM14290 is another lncRNA on chromosome 2 that shares exonic overlap with the gene KCNB1. KCNB1 is a potassium voltage-gated channel subfamily $B$ member 1 that mediates the voltage-dependent potassium ion permeability of 

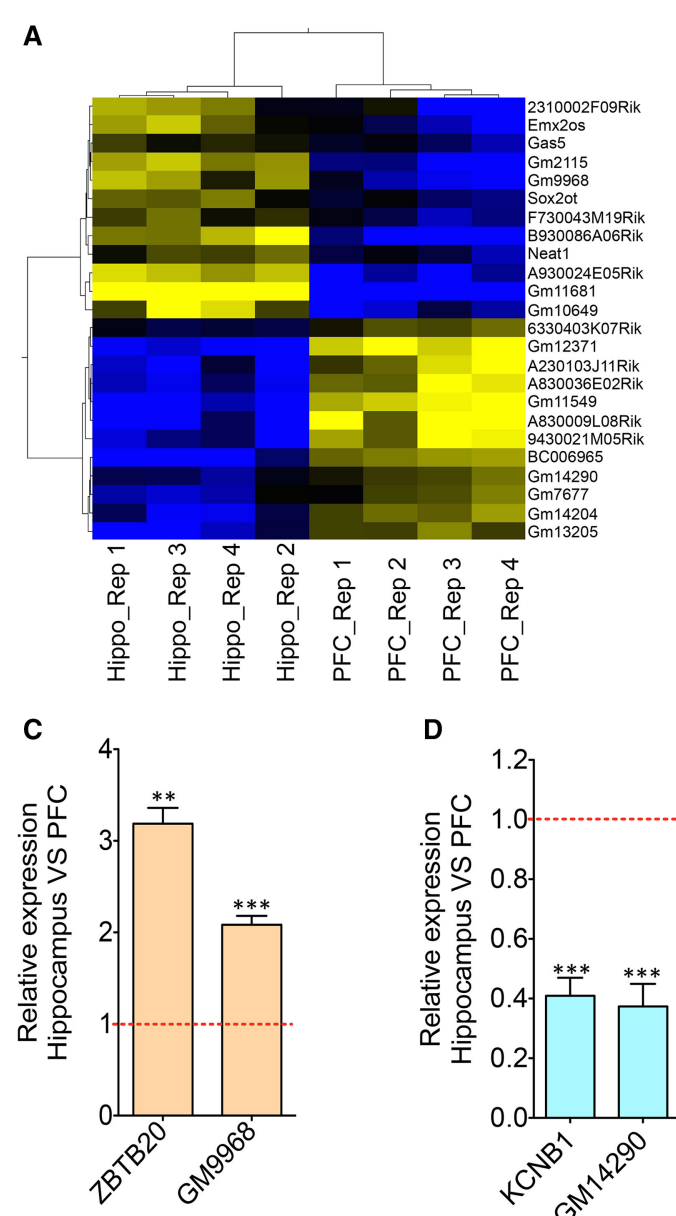

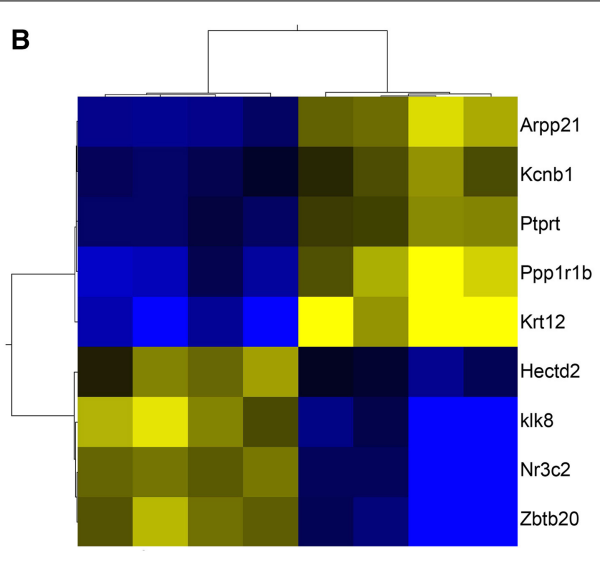

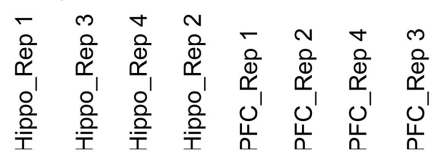

E

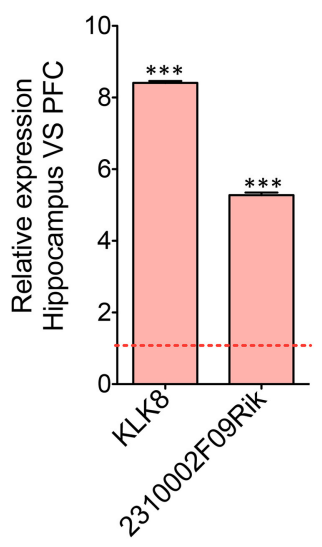

FIGURE 2 | Co-expression analyses of differentially expressed IncRNAs and neighboring mRNAs. (A) Heat map showing differentially expressed IncRNAs between HP and PFC $(N=4)$. (B) Heat map showing mRNAs that are differentially expressed between HP and PFC as well as that present within a $50 \mathrm{~Kb}$ genomic region flanking the genomic locus of differentially expressed IncRNAs in (A). (C-E) Are the qRT-PCR validation of the expression pattern of three IncRNA-mRNA pairs (GM9968 and ZBTB20; GM14290 and KCNB1; 2310002F09Rik and KL8) showing concordant gene expression between the IncRNA and its overlapping mRNA $\left(N=4\right.$; Student $t$-test, ${ }^{* *} P$-value $<0.01,{ }^{* *} P$-value $\left.<0.001\right)$. Data was normalized to 18sRNA level. Red dotted line shows 1-fold change. Error bars represent SEM. excitable membranes. Interestingly, the GM14290-KCNB1 pair is enriched in the PFC compared to the hippocampus and we have confirmed the NGS results with qRT-PCR (Figure 2D). Additionally, our studies showed that 2310002F09Rik is another lncRNA that shares an exonic overlap with $K L K 8$ in the antisense direction. KLK8 (kallikrein-related peptidase 8), known commonly as neuropsin, is a serine protease expressed predominantly in the brain. Earlier analyses using northern blot and in situ hybridization demonstrated that neuropsin is expressed specifically in the limbic system of the mouse brain and is present at the highest concentrations in pyramidal neurons of the hippocampal CA1 and CA3 subfields (Oka et al., 2002).

Consistent with the above studies, our gene expression analyses, both by RNAseq as well as qRT-PCR validated that $\mathrm{klk8} /$ neuropsin is highly enriched in the hippocampus $(\sim 5$-fold difference) compared to the PFC. We found that the lincRNA 2310002F09Rik that shares exonic overlap with neuropsin spans the promoter region of the neuropsin and is enriched in the hippocampus ( $\sim 8$-fold) compared to the PFC. Both genes are enriched very significantly in the hippocampus compared to the PFC. Here, these three pairs had a positive, direct correlation where both mRNA and lncRNA were selectively enriched in either the hippocampus (GM9968-ZBTB20 and 2310002F09Rik-KLK8) or PFC (GM14290-KCNB1). This observation is consistent with the prevalence of concordant expression patterns in mammalian cis-sense or cis-antisense gene pairs (Nakagawa and Kageyama, 2014).

To understand the functional significance of the tissue-specific gene signatures, we applied gene ontology enrichment analysis of upregulated ( $\log 2$ fold change $>1$, adjusted $p$-value $<0.05$ ) and downregulated genes ( $\log 2$ fold change $<-1$, adjusted $p$-value $<$ 0.05) in the hippocampus compared to the PFC using Ingenuity Pathway Analysis (Qiagen-IPA). The top ten upregulated and downregulated pathways were identified and sorted according to 


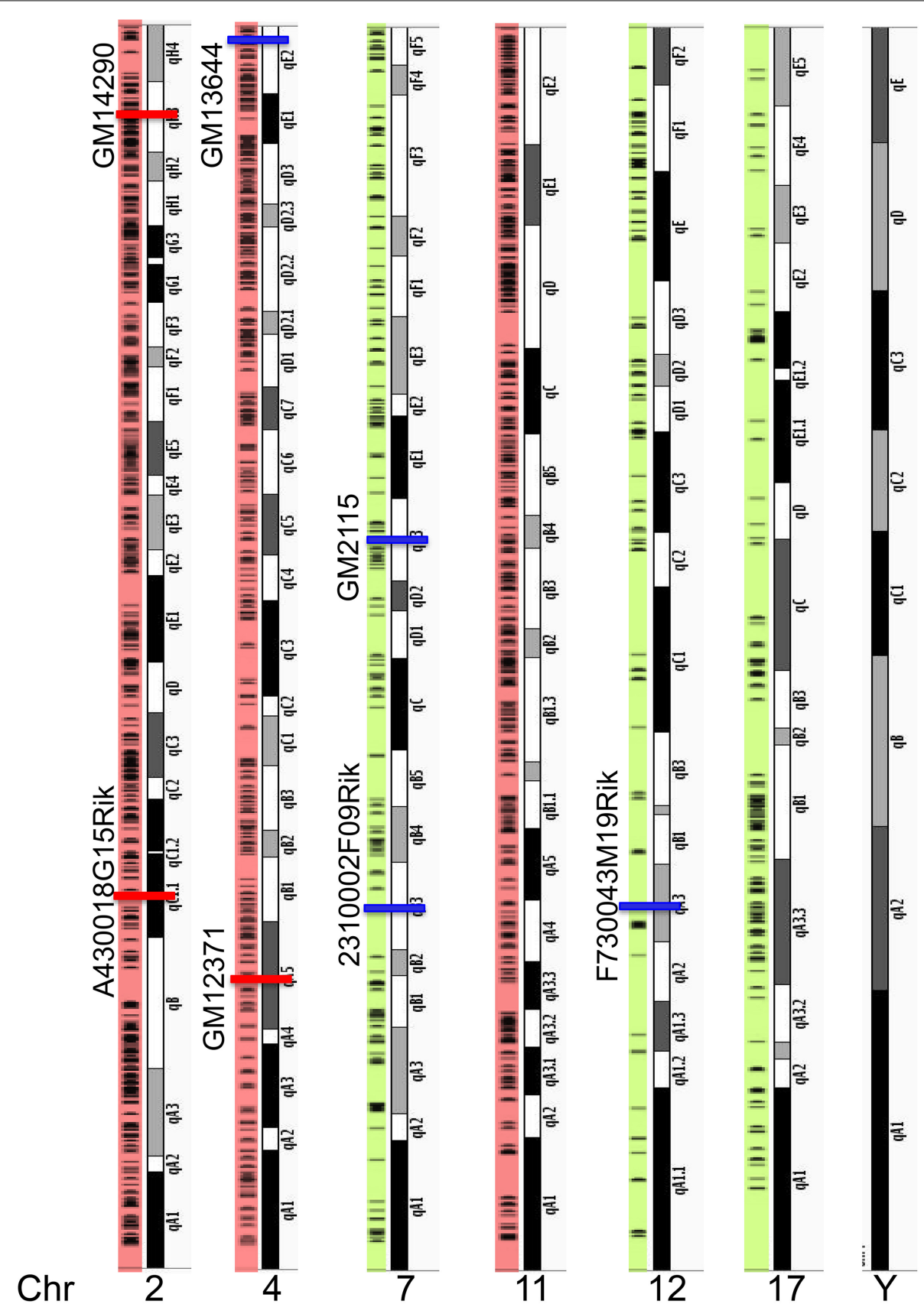

FIGURE 3 | Chromosome locations of all IncRNAs along mouse genome. Top three chromosomes $(2,4$, and 11$)$ that encode maximum number of IncRNAs are highlighted in red and three examples of chromosomes that code less than 150 IncRNAs are highlighted in green. Horizontal black lines in the color shaded along the chromosome show chromosomal location of

individual IncRNAs. This analysis showed clustering of several IncRNAs along

their statistical significance as the negative logarithm of $p$-values calculated by IPA (Figures S4A,B). Our analysis found that many of the differentially expressed mRNAs assigned to the canonical pathway mapping are involved in the categories "Neurological diseases, Psychological diseases and Behavior" and are enriched in the PFC compared to the hippocampus. the chromosomes. All the 21 chromosomes and their IncRNAs distribution are illustrated in Figure S3. Percentage of IncRNAs in each chromosome is provided in Table S2. Chromosomal locations of seven IncRNAs used in this study and their genomic location (Red: enriched in PFC and blue: enriched in $\mathrm{HP}$ ) are indicated in the Figure. None of the IncRNAs we identified in the HP and PFC map onto the $Y$ chromosome.

\section{DYNAMIC EXPRESSION PATTERN OF InCRNAs IN DIFFERENT MOUSE BRAIN REGIONS}

We next studied whether the selectively enriched lncRNAs in the hippocampus and PFC show DE in other regions of the brain (Figure 4A). We have selected 11 lncRNA genes, six of them enriched in the hippocampus and five enriched in the PFC as 


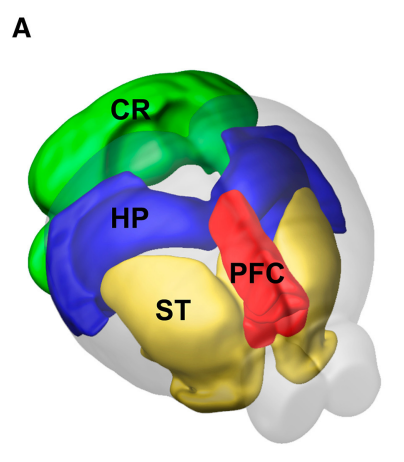

C

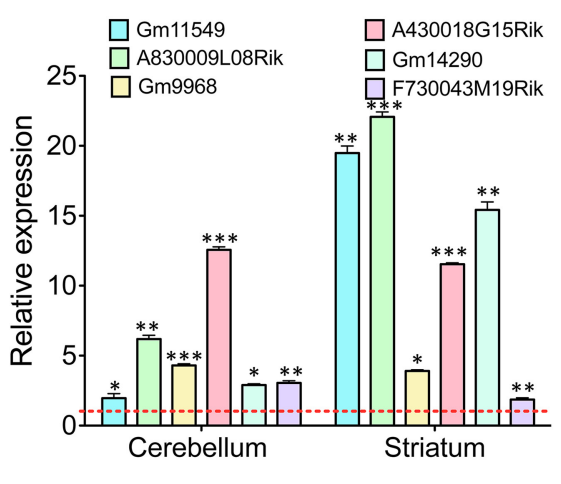

HP Vs Cerebellum \& Striatum

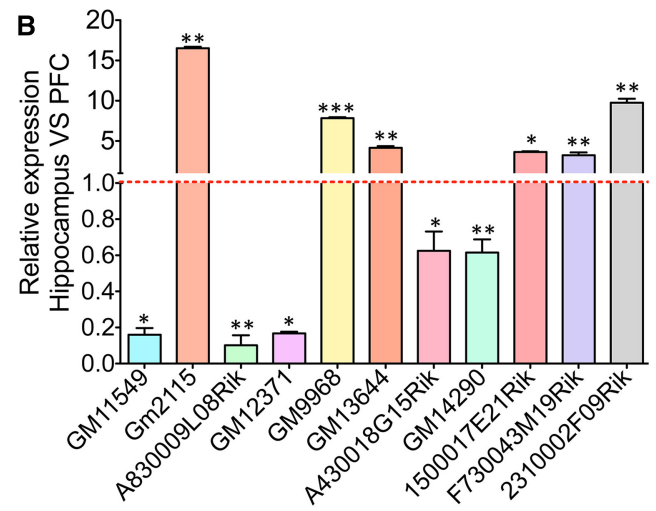

D

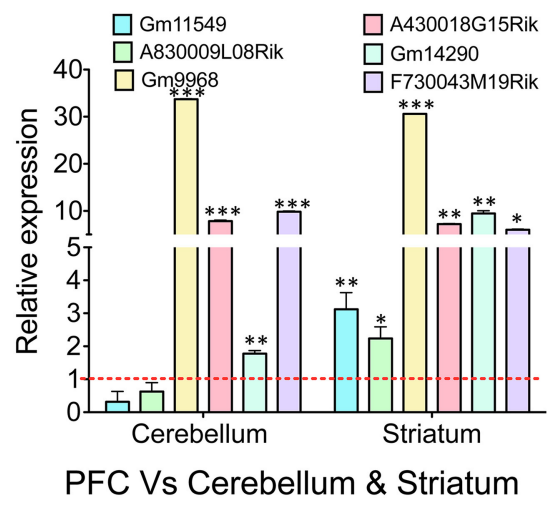

From the list of 11 IncRNAs, we selected six IncRNAs to investigate their expression profile in regions in striatum and cerebellum. (C, D) Show the expression pattern of six IncRNAs in cerebellum and striatum compared to hippocampus and PFC, respectively $(N=4$; Student $t$-test; ${ }^{*} P$-value $<0.05,{ }^{* *} P$-value $<0.01,{ }^{* * *} P$-value $\left.<0.001\right)$. Data was normalized to 18sRNA level. Red dotted line corresponds to 1-fold change. Error bars represent SEM. outlined in Figure 4B and studied their expression in striatum and cerebellum. LncRNAs with a very low expression based on FPKM values were excluded to avoid stochastic biases generated during qRT-PCR amplification. qRT-PCR analyses of these samples were then compared to the RNAseq data for both tissue types.

To corroborate the expression levels measured by RNAseq, the ratio of expression levels between tissues using RNAseq was compared to the ratio of expression as measured by qRT-PCR. For all genes, expression of genes in the hippocampus was measured relative to their expression in the PFC. At first we verified the expression of 11 selected lncRNAs with DE profiles between the hippocampus and PFC based on the $p$-value (adjusted $p$-value $<$ 0.05) from RNAseq analyses (Figure 4B). The hippocampus enriched lncRNA genes were GM2115 (15 \pm 0.2 fold), GM9968 ( $8 \pm 0.12$ fold), GM13644 ( $4 \pm 0.2$ fold), 1500017E21Rik (3.6 \pm 0.1 fold), F730043M19Rik (3 \pm 0.35 fold), and 2310002F09Rik ( $9.7 \pm 0.5$ fold). Three lncRNAs significantly enriched in the PFC were GM11549 (6 \pm 0.1 fold), A830009L08Rik (10 \pm 0.1 fold), and GM12371 ( $6 \pm 0.1$ fold $)$. There were two more lncRNAs, which were only slightly ( $\sim 1.5 \pm 0.2$ fold) but not significantly enriched compared to other lncRNAs described earlier in the PFC (Student's $t$-test; $p<0.05)$. We have compared the fold difference of these $11 \mathrm{lncRNAs}$ obtained from DESeq analysis (Figure S4C) and found that the DE pattern of both qRT-PCR and RNAseq experiments are consistent with each other. Similarly, we studied mRNA genes that are differentially expressed in the hippocampus vs. PFC and found that both qRT-PCR and RNAseq data are in agreement with each other (Figure S4D).

We next analyzed the expression levels of six of these lncRNA in the cerebellum and striatum and compared to the expression levels in the hippocampus and the PFC. We found that most of these selected IncRNAs are differentially expressed between the hippocampus, PFC, cerebellum and striatum [Figure 4C: GM11549 ( $2 \pm 0.1$ and $20 \pm 0.5)$, A830009L08Rik (6.5 \pm 0.3 and $23 \pm 0.4)$, GM9968 (4.5 \pm 0.1 and $4 \pm 0.15)$, A430018G15Rik $(13 \pm 0.25$ and $11.5 \pm 0.1), G M 14290(2.5 \pm 0.1$ and $15 \pm$ $0.7)$, and F730043M19Rik (2.7 \pm 0.15 and $1.75 \pm 0.1)$, Student's $t$-test; $p<0.05]$. In the same way we analyzed the expression fold differences for all six lncRNAs in the cerebellum and striatum, 
respectively, compared to the PFC [Figure 4D: GM11549 (0.3 \pm 0.2 and $3 \pm 0.5)$, A830009L08Rik ( $0.6 \pm 0.26$ and $2.2 \pm 0.35)$, GM9968 (33.7 \pm 0.1 and $30 \pm 0.1)$, A430018G15Rik (7.8 \pm 0.1 and $7.2 \pm 0.2), G M 14290(1.7 \pm 0.1$ and $9.4 \pm 0.57)$, and F730043M19Rik $(9.8 \pm 0.15$ and $6 \pm 0.12)$, Student's $t$-test; $p<0.05]$. These results suggest that different brain regions have characteristic lncRNA expression levels.

\section{InCRNA EXPRESSION IN THE tri-SYNAPTIC CIRCUITRY OF THE HIPPOCAMPUS}

The results from the above experiments suggested that lncRNAs are differentially expressed between brain regions. We next studied how $\operatorname{lncRNAs}$ are differently expressed within a brain region. We first examined the lncRNA expression in dorsal and ventral hippocampus and then in the three specific sub-regions of hippocampus DG (Dentate Gyrus), CA3 (Cornu Ammonis 3), and CA1 (Cornu Ammonis 1)-that constitute the tri-synaptic circuitry. DG, CA3, and CA1 regions contain populations of neurons that are homogeneous and show characteristic electrophysiological properties.

Dorsal and ventral hippocampus are implicated in different cognitive functions (Wang et al., 2013). The in situ hybridization analyses showed the specific distribution of GM9968 lncRNA in dorsal and ventral hippocampus. GM9968 is equally distributed in the DG, CA1, and CA3 regions in the ventral (vHP) as well as dorsal hippocampus ( $\mathrm{dHP}$ ) as seen in Figure 5C. However, between the vHP and dHP, the expression level of GM9968 is different. GM9968 level appears to be higher in the vHP compared to dHP (Figure 5C). On the other hand, GM13644, another lncRNA enriched in the hippocampus, did not exhibit any prevalence in the vHP vs. dHP (Figure 5D). Nonetheless, GM13644 shows DE patterns between CA1, CA3, and DG in both vHP and dHP. From in situ analyses, it is evident that expression of GM13644 is higher in the DG compared to CA1 and CA3 (Figure 5D).

Next, we isolated the subregions of the hippocampus using laser capture microdissection (LCM) and isolated the RNA for qRT-PCR analysis. A representative Cresyl violet staining image showing successful microdissection of CA1, CA3 and DG regions of the hippocampus is shown Figure 5B. The DE analysis of each region compared to the total hippocampus showed that four of the lncRNAs we analyzed follow a unique expression pattern in a specific sub-region of the hippocampus as summarized in Figure 5C. All of these lncRNAs are enriched in these sub-regions compared to the total hippocampus, which suggests potential roles in spatial regulation of gene expression of lncRNAs. Additionally, we found differences in the expression levels of some of these lncRNAs between CA1, CA3, and DG. For example, GM2115 is significantly enriched in the CA1 relative to both CA3 and DG (Student's $t$-test, $p<0.05$ ) whereas CA3 and DG have similar levels (Figure 5E). In the case of 2310002F09Rik, CA1 has significantly lower expression levels (Student's $t$-test, $p<$ 0.05 ) relative to both CA3 and DG (Figure 5E). Another lncRNA, GM13644, is significantly enriched in the CA3 and DG compared to CA1 (Student's $t$-test, $p<0.05$; Figure 5E). These differences in the expression levels indicate neuron specific expression profile of lncRNAs within subregions of the hippocampus. As one of the most abundantly expressed lncRNAs in the hippocampus and
PFC, MALAT-1 did not show any preferential expression between these two regions, and within the hippocampal subregions.

qRT-PCR results showed lncRNAs we studied in DG and CA3 have similar expression levels, and expression in CA1 is lower than that in DG and CA3. The slight disparity could come from the challenges posed by LCM during which some amount of RNA degradation unavoidably occurs even under the most stringent RNase-free environment, as well as from the following linear amplification (Wang et al., 2010), where aRNA production leads to shortening of transcripts over successive cycles of amplification (Clement-Ziza et al., 2009). These results indicate a unique spatial distribution of specific lncRNAs within the same anatomical region of the adult mouse brain.

It has been shown that the lncRNAs are trafficked to specific subcellular locations (Prasanth et al., 2005; Puthanveettil et al., 2013; Kadakkuzha et al., 2014; Van Heesch et al., 2014), which are directly indicative of their function. We next investigated the subcellular distribution of four different lncRNAs in the CA1, CA3, and DG neurons and CG1 (cingulate cortex 1) region of PFC. For CA1, CA3, and DG neurons, we selected GM9968 and GM13644, which we analyzed earlier for hippocampal subregion specific expression. To analyze the specific subcellular localization in the PFC, we selected two lncRNAs, GM11549 and A830009L08Rik that are enriched in the PFC, relative to the hippocampus (Figure 4A). We found that all four lncRNAs were enriched in the nucleus compared to the cytoplasm (Figures 6A-D). In order to validate the subcellular distribution of lncRNAs observed from in situ, we purified RNA from nuclear and cytoplasmic fractions from the hippocampus and PFC followed by qRT-PCR. Figures $6 \mathrm{E}, \mathrm{F}$ indicate that the qRT-PCR data is in agreement with the in situ hybridization analyses and show prevalence of nuclear localization by lncRNAs.

\section{DISCUSSION}

A recent study of a few highly conserved and brain-expressed mouse lncRNA transcripts and their orthologs in birds and marsupial found that unlike protein-coding genes, the sequences, exon structures, and transcriptional start sites for these noncoding genes are all highly variable. Similar large-scale studies have discovered fundamental characteristics of lncRNAs including their low levels of expression, temporal and spatial patterns of expression exons junction modulation, and disease-related splicing events. In a recent RNA-Seq study in Parkinson Disease (PD) patients that sequenced libraries from leukocytes and two brain regions added over 7000 lncRNAs to the human lncRNA database, of which 3495 were co-expressed in the PD brain and leukocytes suggesting the involvement of lncRNA in neurodegenerative diseases (Soreq et al., 2014). Our results and the above mentioned study outcomes are providing framework to find out the diverse mechanisms through which lncRNAs act to regulate protein-coding genes at both the transcriptional and translational levels.

Estimates from the most recent GENCODE project in mouse and humans indicate the presence of 4074 and 13,870 lncRNAs, respectively. Unlike the numbers of protein coding genes, which do not increase with organism complexity, the numbers of lncRNAs increase significantly (Khaitovich et al., 2006). The 
A

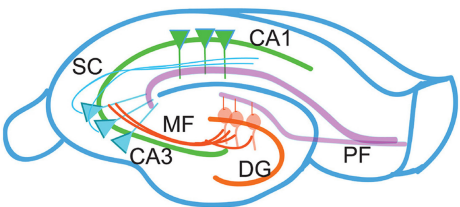

B

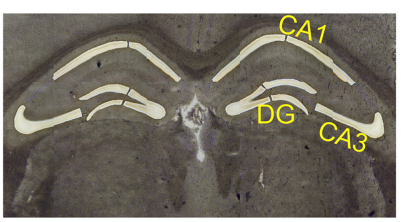

C

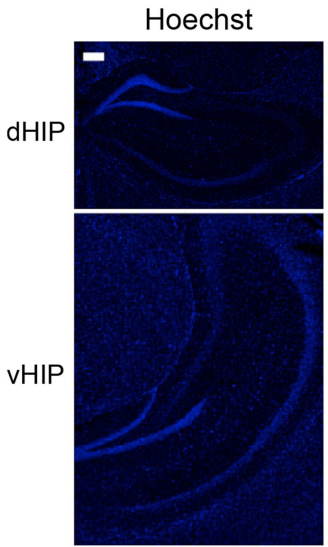

D

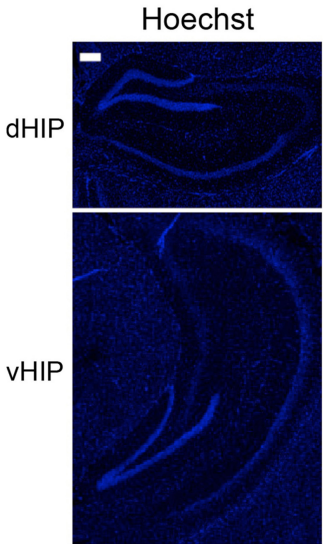

E

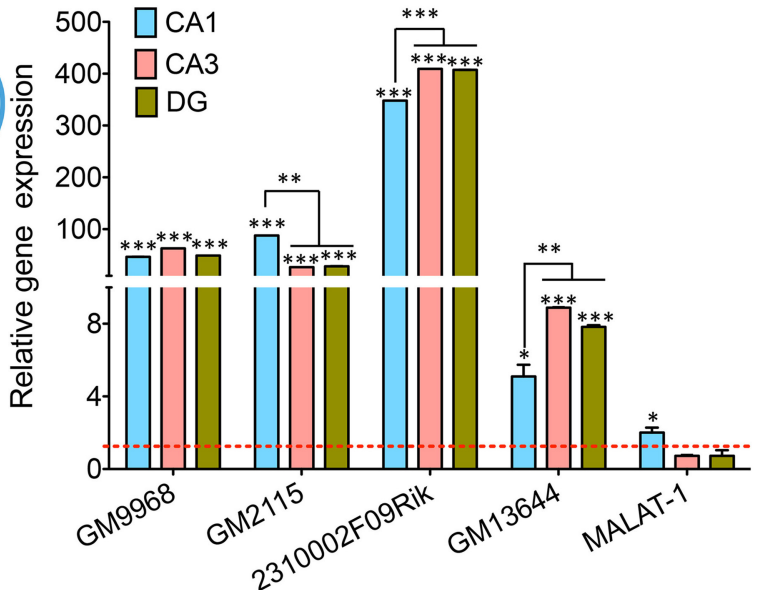

GM9968

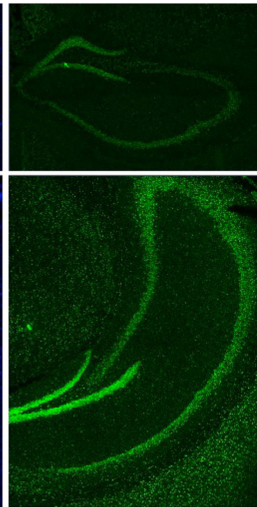

GM13644
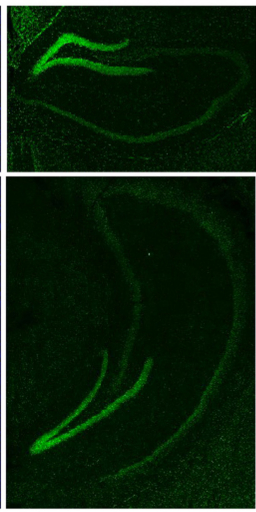

$\beta$-tubulin

Merged
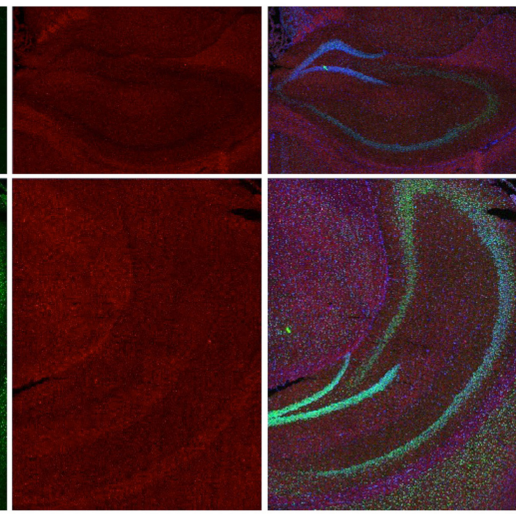

$\beta$-tubulin

Merged
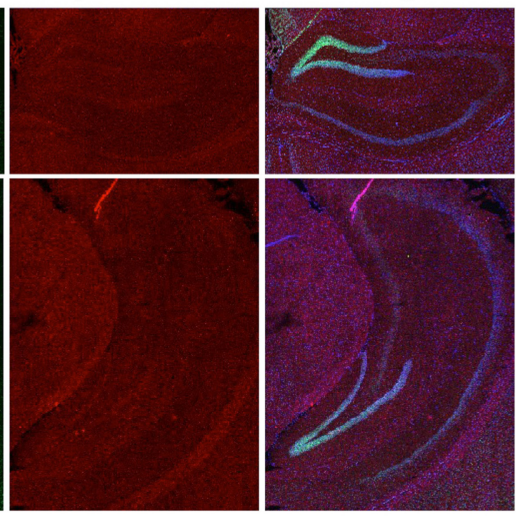

FIGURE 5 | Subregion specific expression of IncRNAs in the

hippocampal tri-synaptic circuitry. (A) Cartoon of the tri-synaptic circuitry of the hippocampus showing the CA1, CA3, DG sub regions and synaptic connections between DG and CA3 and CA3 and CA1. (DG: dentate gyrus; PF: Perforant path, MF: Mossy fibers and SC: Schaffer collaterals). (B) $16 \mu \mathrm{m}$ coronal sections of the mouse brain, stained with Cresyl violet showing successful laser capture microdissection of CA1, CA3, and DG regions of the mouse brain for qRT-PCR analysis of IncRNA expression. (C, D) Are the confocal projection images of coronal section of hippocampus showing expression IncRNAs GM9968 and GM13644 in dorsal and ventral hippocampus (dHP: dorsal hippocampus; vHP: ventral hippocampus). Both IncRNAs (both green) co-stained with $\beta$-tubulin protein (red), along with the nuclear stain Hoechst (blue) and the merged images are shown. Scale bar, $200 \mu \mathrm{m}$. (E) qRT-PCR analysis of the five IncRNAs enriched hippocampus in the CA1 CA3, and DG subregions. CA1, CA3, and DG were microdissected (B) using LCM. The dotted red line shows 1-fold enrichment. Data was normalized to 18sRNA levels. Bar graphs show enrichment of five IncRNAs (indicated in the Figure) in the three subregions compared to the entire hippocampus $\left(N=4\right.$; Student $t$-test, ${ }^{*} P$-value $<0.05,{ }^{*} P$-value $<0.01$, ${ }^{* * *} P$-value $\left.<0.001\right)$. Error bars represent SEM. 


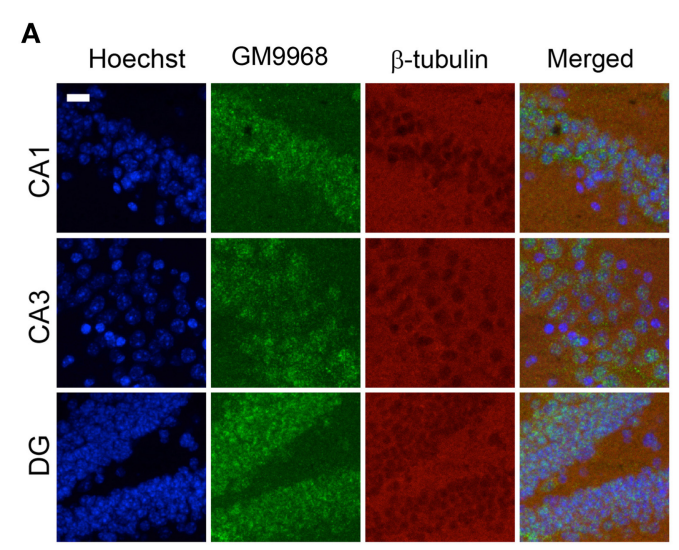

C

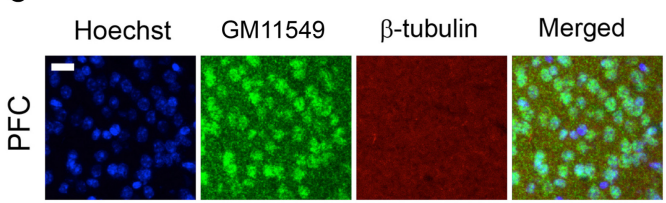

D

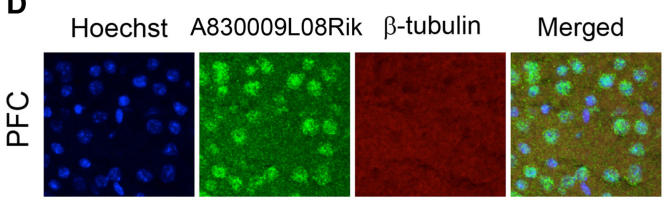

B

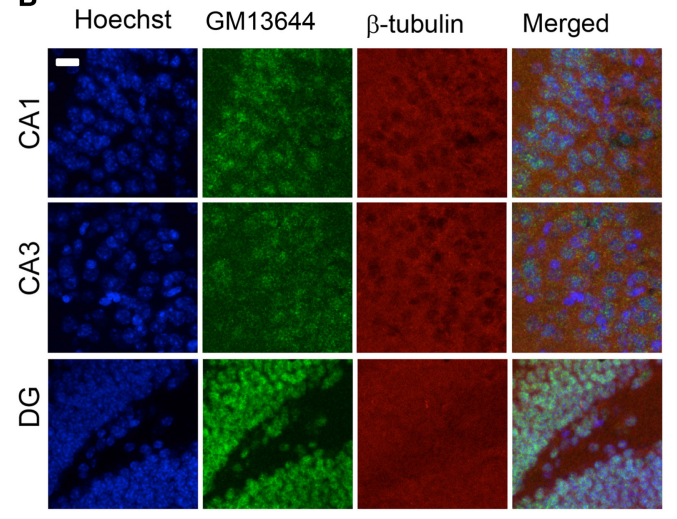

E

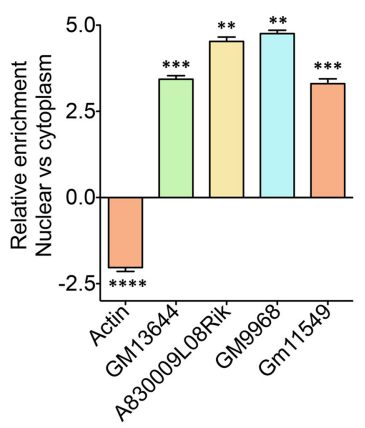

$\mathbf{F}$

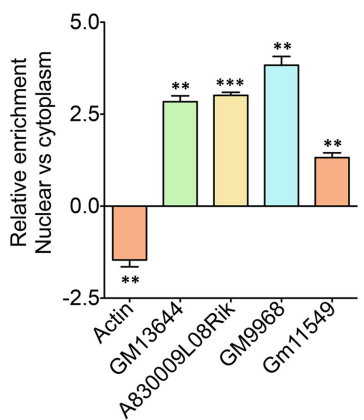

FIGURE 6 | Subcellular localization of IncRNAs in hippocampus and PFC. Confocal projection images of coronal section of the hippocampus $(\mathbf{A}, \mathbf{B})$, and PFC (C,D) show in situ hybridization of two IncRNAs (indicated in the Figure) along with immunohistochemical analysis of $\beta$-tubulin. The respective IncRNA is in green co-stained with $\beta$-tubulin protein (red), along with the nuclear stain Hoechst (blue). Images in (A,B) show the subcellular localization of IncRNAs GM9968 and GM13644 in the CA1, CA3, and DG subregions of the tri-synaptic loop, respectively. (C,D) show the subcellular localization of IncRNAs GM11549 and A830009L08Rik in the PFC. Scale bar, $20 \mu \mathrm{m}$. (E,F) qRT-PCR validation of GM9968, GM13644, GM11549, and A830009L08Rik transcripts in the nuclear vs. cytoplasmic fractions from hippocampus and PFC. Relative enrichments of IncRNAs were calculated using delta (deltaCT) values where CT values were first normalized to GM11549 to get deltaCT. $\beta$-actin was used as a positive control to validate effective fractionation. Error bars are SEM. vast difference in the number of lncRNAs between human and mouse is intriguing as both species have a similar number of protein coding genes, and implies the role of lncRNAs in evolutionary development. Confirming this notion, it has been reported that that changes in lncRNA sequences constitute half of all the genetic differences between the human and chimpanzee genomes (10). However, it has yet to be determined if these changes are causative of the differences between humans and chimpanzees or if they are the result of genetic drift (Carninci et al., 2005). Despite their significance little is known about the functions and identity of lncRNAs in specific brain regions and specific neuronal populations of the vertebrate brain. Specifically, we do not have unbiased estimates of populations of lncRNAs in sub-regions of the vertebrate brain. To address this, we have carried out RNAseq analysis of the total transcriptome to identify lncRNA population in two key regions of the brain, hippocampus, and PFC of the adult mouse. Annotation of lncRNAs in the mouse genome is yet to be completed and is often challenging due to the low expression levels of these transcripts, which leads to poor definition of the transcript boundaries.
To compare the overall expression status of lncRNAs and mRNAs in the hippocampus and PFC, we employed whole transcriptome sequencing and identified a total of 20,618 genes in the hippocampus and 20,025 in the PFC, including 2759 lncRNAs in the hippocampus and 2561 lncRNAs in the PFC. Less restrictive values for compared methodologies usually results in far too large a number of selected genes and here we applied a rather conservative adjusted $P$-value of 0.05 instead of $P$-value to identify the lncRNAs in these two regions. These lncRNA transcripts reported from the present study, out of the 3823 annotated lncRNA transcripts in the mouse genome, according to NCBIM37, represent a very robust $(\sim 70 \%)$ expression pattern of lncRNAs in the adult mouse brain. Consistent with the previous results that lncRNAs are generally expressed at lower levels than protein-coding genes and are more likely to display tissue-specific pattern of expression (Mercer et al., 2008; Derrien et al., 2012; Kutter et al., 2012), based on FPKM values we find that more than $80 \%$ of lncRNAs are expressed much lower than mRNAs (Figure S1) present in the same brain regions. Our depth of sequencing restricted us from identifying novel lncRNAs and recently described circular 
non-coding RNAs such as competing endogenous RNAs (Jeck et al., 2013).

Consistent with previous reports (Cabili et al., 2011; Derrien et al., 2012), we found that most of the lncRNA expressed (53\%) belong to the lincRNA category followed by antisense RNAs and a low prevalence of other biotypes in both the hippocampus and PFC. We next mapped all the lncRNAs from the hippocampus and PFC onto mouse chromosomes and found that they are present as clusters in varying numbers along the chromosomes, with three chromosomes (Figure S3: 2, 4, 11) expressing $>300$ lncRNAs and the rest of the chromosomes contained loci for 50-150 lncRNAs. These results are consistent with previous finding on mRNAs and lncRNA pairs in the developing mouse brain that a large number of brain-expressed lncRNA loci are not evenly distributed along the mouse chromosomes (Ponjavic et al., 2009).

We next searched for mRNAs and miRNAs within the 50 $\mathrm{Kb}$ of loci of differentially enriched lncRNAs to examine possible co-regulation of expression. We identified three lncRNAmRNA pairs [GM9968 and ZBTB20; 2310002F09Rik and KLK8 (neuropsin); GM14290 and KCNB1] that displayed concordant expression. The observation that these three pairs of tightly co-expressed, share common genomic loci, and are differentially expressed between the hippocampus and PFC, suggest that these lncRNAs might regulate neuronal functions mediated by these mRNAs. For example, the lncRNA 2310002F09Rik, which is enriched in the hippocampus and the protein-coding gene KLK8/neuropsin, share a $5^{\prime}$ exonic overlap in the antisense direction (Figure 7A). Neuropsin is key in hippocampal plasticity (Oka et al., 2002) and plays an important role in brain diseases, such as Alzheimer's disease and Epilepsy. Another example is GM9968, an lncRNA that is significantly enriched in the mouse hippocampus. GM9968 completely overlaps with ZBTB20 in the antisense direction (Figure 7B). Previous studies showed that ZBTB20 in mature CA1 plays an important role in LTP and memory by regulating $N M D A R$ activity, and activating $E R K$ and $C R E B$ in mice (Ren et al., 2012). In addition, hypermethylation in the ZBTB20 gene is shown to be associated with major depressive disorders. Taken together, these observations suggest that by regulating expression of specific mRNAs these lncRNAs might engage in neural plasticity and neuropsychiatric disorders.

Focusing on a subset of lncRNAs, we find that different brain regions and neuronal populations have characteristic lncRNA expression profiles. We examined expression differences in striatum, cerebellum, PFC, hippocampus and differences between dorsal and vHP and within the well-studied tri-synaptic circuitry of the hippocampus. The mammalian brain harbors a large variety of neuron types and sub-types, arranged in specific layers within the sub-regions. The hippocampus is an intensively studied part of the brain with dedicated sub-regions that differ in their physiological functions and capacities and display quite a specialized response to pathophysiological stimuli as illustrated in neurodegenerative disorders. Our studies showed an interesting distribution of these lncRNAs in the tri-synaptic circuitry. The two hippocampus enriched lncRNAs, GM9968 and GM13644, showed different expression patterns. GM9968 is equally distributed in the DG, CA1, and CA3 regions, but enriched in the vHP compared to the dHP. GM13644 on the other hand, is equally distributed in the vHP and $\mathrm{dHP}$, but highly enriched in DG compared to CA1 and CA3. Thus, lncRNAs show DE along dorsal-ventral axis of the hippocampus (Figure 5). Consistent with these observations we find that specific neuronal populations in hippocampus have characteristic lncRNA expression profiles. Our in situ hybridization and sub-cellular fraction analysis add another layer to the complexity of lncRNA expression. These studies suggest that lncRNAs could be enriched in specific cellular

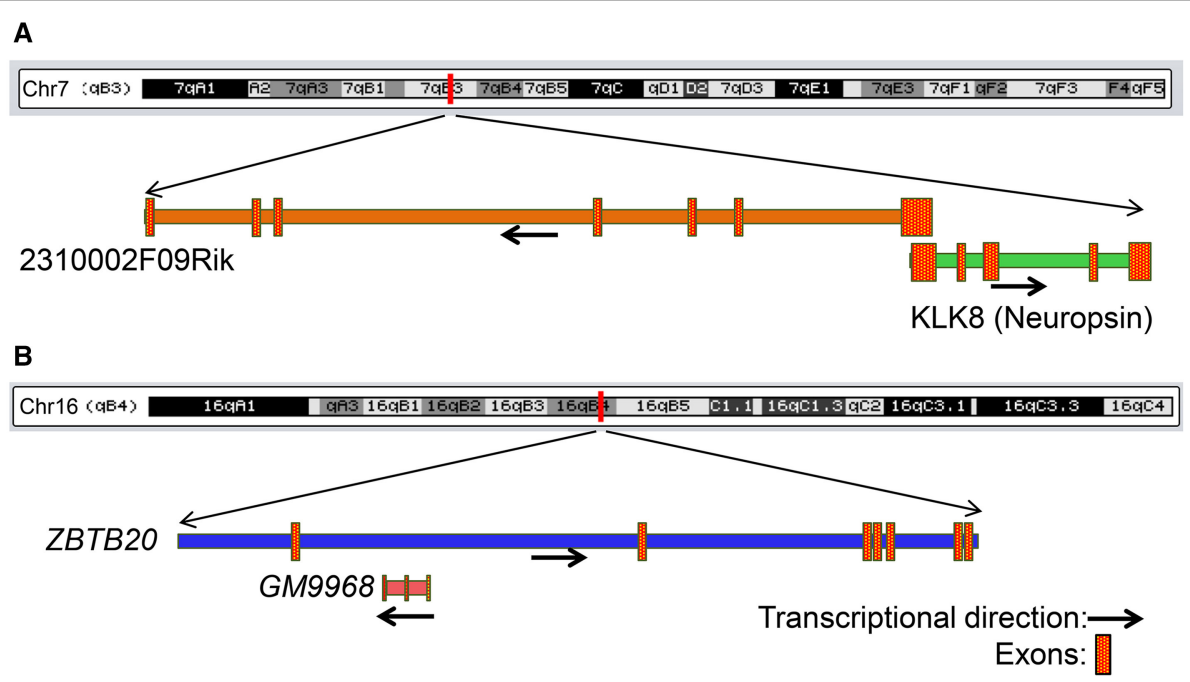

FIGURE 7 | Genomic context of two IncRNAs that share genomic loci with protein-coding genes associated with neurological diseases. (A) Genomic locus of the mRNA KLK8 (neuropsin) and IncRNA 2310002F09Rik on chromosome 7. KLK8-2310002F09Rik pair has a $5^{\prime}$ exon overlap and both genes are enriched in the hippocampus. KLK8 is implicated in Epilepsy and
Alzheimer disease. (B) Genomic locus of the mRNA ZBTB20 and IncRNA GM9968 on chromosome 16. GM9968 completely overlap with ZBTB20 in antisense direction. ZBTB20 is implicated in major depressive disorder. The arrow represents the direction of transcription and the boxes represent exons. 
compartments. Taken together, the tissue-specific expression patterns and the distinct subcellular localization of many lncRNAs suggest that their expression is under precise control. We now provide novel details on the expression patterns of lncRNAs per hippocampal sub-region and are of potential interest in further investigation of how lncRNAs regulate gene expression and other hippocampus dependent functions such as learning and memory.

In summary, in this study, we provide the first unbiased estimates of lncRNAs in two key brain regions, the hippocampus and PFC that are involved in memory and cognition. We have characterized their expression, in detail, including their chromosome distribution, genomic context, sub-region specific analyses and subcellular distribution. Our analyses establish complex lncRNA expression profiles in the mouse brain. This DE might play important roles in the development, physiology, plasticity and pathology of the mammalian brain. These findings provide a foundation for further research into the molecular mechanisms of lncRNAs in mammalian brain functions.

\section{MATERIALS AND METHODS ANIMALS}

7-9 week old male C57Bl/J6 mice (Jackson Laboratories) were housed in groups of four on a $12 \mathrm{hr}$. Light/dark cycle with ad libitum access to food and water. All experiments were performed during the light part of the diurnal cycle. Housing, animal care and experimental procedures were consistent with the Guide for the Care and Use of Laboratory Animals and approved by the Institutional Animal Care and Use Committee of the Scripps Research Institute.

\section{RNA PREPARATION FOR NEXT GENERATION SEQUENCING (NGS)}

A total of 8 mice were sacrificed and the respective brain tissues from two mice each were pooled together, providing the sample size of 4 for NGS. 8-12 week old male C57Bl/J6 mice (Jackson Laboratories) were sacrificed and the brain was removed from the skull and placed in ice-cold HEPES HBSS (HHBSS: $1 \times$ Hank's basal salt solution, $2.5 \mathrm{~mm}$ HEPES-KOH, pH 7.4, $35 \mathrm{~mm}$ glucose, and $4 \mathrm{~mm} \mathrm{NaHCO} 3$ ). The hippocampus and PFC was dissected and homogenized in Trizol using a glass-Teflon homogenizer containing $1 \mathrm{ml}$ of ice-cold Trizol (Life technologies, USA). RNA was extracted from each fraction using the Trizol-Chloroform method (Life technologies) and stored at $-80^{\circ} \mathrm{C}$ until used.

\section{NEXT GENERATION SEQUENCING AND DATA ANALYSIS}

Beginning with a $200 \mathrm{ng}$ total RNA sample, following the removal of ribosomal RNAs, the sample was reverse transcribed with random hexamer and poly A primers. This product was converted into double stranded cDNA, followed by SPIA amplification. After purification the sample was end repaired and then had specific adapters ligated to the $5^{\prime}$ and $3^{\prime}$ ends. These products are then PCR amplified to enrich for the ligated products (Nugen San Diego, CA). The amplified samples were then purified and diluted down to a $10 \mu \mathrm{M}$ library. This library was then treated with $\mathrm{NaOH}$ to produce single stranded amplicons, which were then added to the Illumina $\mathrm{cBOT}$ instrument to generate the double stranded clusters used for the massively parallel sequencing on the Illumina
Genome Analyzer IIx according to manufacturer specifications. The read length used for RNA seq is $75 \mathrm{bp}$.

Analysis of the RNAseq data was performed using Tophat2 (v.2.0.9) (Kim et al., 2013) and the Cufflinks suite (v.2.1.1) (Trapnell et al., 2010). Trimming of the data was performed using the FastX-Toolkit (v.0.0.13) $\left(\right.$ Toolkit $\left.^{1}\right)$. The reads were trimmed using the quality scores (cut-off Phred score $=28$ ). The trimmed reads were aligned to the Mus musculus genome build NCBIM37 using Tophat. Tophat was run using its default parameters, which include a maximum of 2 mismatches, a maximum gap length of 2 gaps and an edit distance of 2 for the final alignment.

After the alignment, Cufflinks was utilized to calculate the FPKM values. The default parameters were also used to run cufflinks, except for one parameter "-max-bundle-frags" that was changed to 100,000,000. Cufflinks measure transcript abundances in Fragment Per Kilobases of transcripts per million fragments mapped. The alignment data were then processed and quantified using HTSeq (Anders et al., 2015). The raw read counts generated in HTSeq were used to identify differentially expressed genes in the R Bioconductor package DESeq (Anders and Huber, 2010). HTSeq count was run with "-stranded = no" option, and the ID attribute option, "-i" set to "gene_name." DESeq uses the total size of each library to normalize the raw read counts and perform calculations on fold change as well as significance based on $p$-value and adjusted $p$-value. The comparisons in this case are between samples of two groups, hippocampus and PFC. The distance matrix analysis based on DESeq method indicates that the hippocampus samples and PFC samples cluster together (Figure S2). LncRNA transcripts were extracted from the Ensembl annotation file (NCBIM37) and categorized into eight biotypes.

\section{CO-EXPRESSION ANALYSIS/IPA/CHROMOSOME DISTRIBUTION}

Twenty-four differentially expressed long non-coding RNAs (adjusted $p$-value $<0.05$ ) were selected for co-expression analysis. At first, all the protein coding genes flanking each lncRNA within $50 \mathrm{~Kb}$ chromosomal region was selected from the UCSC genome browser. A total of nine neighboring differentially expressed protein-coding genes within $50 \mathrm{~Kb}$ distance from these lncRNAs were identified using the UCSC genome browser. To generate heat maps of these two corresponding gene lists (Figures 2A,B), the GeneSpring GX V12.1 (Agilent Technologies) two dimensional hierarchical clustering algorithm was applied to median centered DESeq $\log 2$ normalized read counts (yellow/black/blue indicate higher, median and lower expression, respectively). The similarity measure was set to Pearson centered and the linkage rule was set to average.

Ingenuity Pathway Analysis software (Qiagen-IPA) was used to identify enriched canonical pathways in the list of up-regulated (log2 fold change $>1$, adjusted $p$-value $<0.05$ ) and downregulated genes ( $\log 2$ fold change $<-1$, adjusted $p$-value $<0.05$ ) in the hippocampus compared to PFC. The top 10 up-regulated and down-regulated pathways were identified and sorted according to their statistical significance as the negative logarithm of $p$-values calculated by IPA.

\footnotetext{
${ }^{1}$ http://hannonlab.cshl.edu/fastx_toolkit/index.html by Hannon Lab.
} 
A karyotype displaying the location of all lncRNA transcripts was prepared using IGV (Integrated Genomics Viewer) (Robinson et al., 2011). The loci of lncRNAs were obtained from cufflinks and this information was used to create a BED file. This BED file, along with the karyotype from reference genome $\mathrm{mm}$, was used to create the karyotype of lncRNA transcripts.

\section{QUANTITATIVE REAL-TIME PCR (qRT-PCR)}

RNA was isolated from the tissues dissected from the adult mouse brain as described earlier and cDNA was generated by reverse transcription as described earlier (Kadakkuzha et al., 2013). Briefly, $1 \mu \mathrm{g}$ of RNA was used with Quanta cDNA SuperMix (Quanta Biosciences, Gaithersburg, MD) according to the manufacturer's instructions and the expression of transcripts were quantified by qRT-PCR using SYBR Green PCR master mix (Applied Biosystems Carlsbad, CA) for detection in ABI 7900 cycler (Applied Biosystems Carlsbad, CA). All the qPCR amplifications were performed in quadruplicate in a total volume of $10 \mu \mathrm{l}$ containing $2 \mu \mathrm{l}$ of $\mathrm{H} 2 \mathrm{O}, 2 \mu \mathrm{l}$ of cDNA, $5 \mu \mathrm{l}$ of $2 \mathrm{X}$ Master Mix, $1.0 \mu \mathrm{l}$ of $10 \mu \mathrm{M}$ (each) forward and reverse primers. Quantification of each transcript was normalized to the mouse $18 \mathrm{~S}$ reference gene following the $2^{-\Delta \Delta \mathrm{Ct}}$ method (Livak and Schmittgen, 2001). Student $t$-test was used to select genes with statistically significant expression levels where ${ }^{*} p$-value $<0.05$, ${ }^{* *} p$-value $<0.01,{ }^{* * *} p$-value $<0.001$. The sequences of primers for the mRNAs and lncRNAs are given in the Table S3.

\section{DISSECTION AND FREEZING OF MOUSE BRAINS FOR IN SITU HYBRIDIZATION}

7-9 week old C57BL/6J male mice were used here. Mice were sacrificed and the brain was removed from the skull and quickly washed in ice-cold HEPES HBSS (HHBSS: $1 \times$ Hank's basal salt solution, $2.5 \mathrm{~mm}$ HEPES-KOH, pH 7.4, $35 \mathrm{~mm}$ glucose, and $4 \mathrm{~mm} \mathrm{NaHCO} 3$ ) containing $100 \mu \mathrm{g} / \mathrm{ml}$ of cycloheximide, protease inhibitors (1 tablet $/ 50 \mathrm{ml}$, complete EDTA-free from Roche Products, Hertfordshire, UK) and SUPER•aseIn ${ }^{\mathrm{TM}}$ RNase Inhibitor $(1 \mathrm{U} / \mu \mathrm{l})$, Life Technologies, Grand Island, NY). Then, brains were embedded in OCT FSC 22 Blue frozen section compound (Leica Biosystems, Richmond, IL, USA) and immediately frozen at $-80^{\circ} \mathrm{C}$.

\section{CRYOSECTIONING}

The fresh, frozen brains were sectioned at $16 \mu \mathrm{m}$ on a Leica $3050 \mathrm{~s}$ cryostat. This thickness was optimal for minimizing sectioning artifacts, and was adequate for probe penetration into the section during the in situ hybridization procedure. Each OCT block containing a fresh, frozen brain was trimmed in the cryostat until reaching the desired starting section. Two brains sectioned in coronal plane were used to generate 3 series of 24 slides each. Each series contained sections collected from the same brain area (dHP, vHP, and PFC) and each slide of a given series contained four $16 \mu \mathrm{m}$ thick sections ( 2 slices per brain).

\section{IN SITU HYBRIDIZATION}

DIG labeled ribo probes complementary to selected lncRNAs for in situ hybridization were prepared by in vitro transcription of cDNA templates by using T7 RNA polymerase as described earlier (Kadakkuzha et al., 2014). Briefly 400 nt long sense strand of each transcript was prepared by PCR using mouse hippocampus cDNA as a template and transcript specific PCR primers (Table S4) and ligated to pCRII-TOPO Vector with dual promoters T7 and SP6 (Invitrogen, Cat. Number K4600-01). The Vector with the sense IncRNA DNA in the 5-3' direction was linearized with BamH I (New England Biolab) for transcription using T7 RNA polymerase to generate antisense probes. A small aliquot $(2 \mu \mathrm{l})$ was run on $1.5 \%$ agarose gel to confirm the integrity of RNA probes and stored at $-80^{\circ} \mathrm{C}$ until used. The in situ hybridization using digoxigenin (DIG) labeled riboprobes was followed as described in Puthanveettil et al. (2013). Fifteen $\mu$ M Cryo-sections were fixed in a freshly prepared solution of $4 \%$ paraformaldehyde for $15 \mathrm{~min}$ at room temperature, washed three times for $5 \mathrm{~min}$ in $0.01 \mathrm{M}$ PBS ${ }_{\text {DEPC }}$ and acetylated in freshly prepared TEA solution for $10 \mathrm{~min}$ and pre-hybridization at $68^{\circ} \mathrm{C}$ overnight. After hybridization, the antisense RNA probes were visualized using a Fluorescent Antibody Enhancer kit (Roche, Basel, Switzerland) for DIG detection. The immunohistochemical analyses of btubulin were performed for each slice. Alexa Fluor ${ }^{\circledR} 546$ Donkey Anti-Mouse IgG secondary antibodies (Life Technologies) were used for visualization. Images were acquired by using Zeiss LSM 780 confocal microscope system with $20 \mathrm{X} / 63 \mathrm{X}$ objective. Only projection images are shown.

\section{LASER CAPTURE MICRODISSECTION (LCM)}

7-9 week old C57BL/6J male mice brains were freshly frozen in OCT compound and sectioned at $14 \mu \mathrm{M}$ on Leica $3050 \mathrm{~s}$ cryostat, taking every other section throughout the hippocampus. Sections were then mounted on PEN membrane slides, $\sim 12$ sections per slide with 3 slides per brain. The staining and preparation of sections for the LCM procedure was done using the LCM staining kit (Cat\# AM1935, Life Technologies) with Cresyl Violet following manufacturer's recommendations. Following staining, slides were kept at room temperature before beginning LCM. The microdissection of the CA1, CA3 and DG hippocampal regions were done using the Arcturus PixCell II LCM microscope where the laser power was set at $50 \mathrm{~mW}$ and each capture was done with 2 or fewer laser pulses. After dissection, each sample was placed in $50 \mu \mathrm{l}$ ice-cold Trizol and RNA was extracted using the TrizolChloroform method (Life technologies). Purified RNA from each dissection was subjected to a single round of linear T7 RNA Polymerase-driven transcription using MessageAmp ${ }^{\mathrm{TM}}$ II aRNA Amplification Kit (Cat\# AM1751, Life Technologies).

\section{CYTOPLASMIC AND NUCLEAR SEPARATION OF InCRNAs FROM PFC AND HIPPOCAMPUS}

PFC and hippocampi of two adult mice were used to extract the total RNA. The tissues were dissected from the whole brain and briefly washed in 1X PBS and immediately transferred into a mortar containing liquid nitrogen to cover the sample and to thoroughly grind the tissues. After the grinding was complete, the liquid nitrogen was allowed to evaporate off without allowing the tissues to thaw. Cell lysis and extraction of cytoplasmic and Nuclear RNA were carried out using SurePrep, Nuclear or Cytoplasmic RNA Purification Kit (Cat. \# BP280525, Fisher Bioreagents) according to the kit protocol (Jiang et al., 2014). cDNAs were generated from the purified RNA by reverse 
transcription using Quanta cDNA SuperMix as described earlier and the expression levels of each gene were determined by qPCR using SYBR Green PCR master mix as described earlier. CT values of each lncRNA were first normalized to GM11549, resulting in a deltaCT value. Relative differences between lncRNAs and GM11549 were determined by subtracting the delta CTs of cytoplasm fraction from the delta CTs of nucleus fraction for each sample, resulting in delta (deltaCT) values (Van Heesch et al., 2014). $\beta$-actin mRNA that is enriched cytoplasm was used as a control to validate the efficacy of nuclear and cytoplasmic fractionation.

\section{AUTHOR CONTRIBUTIONS}

By performed RNA sequencing; MF, GS, and ACC performed the RNAseq data analyses; BMK, XL, VR, BR, and SVP designed the experiments. BMK, XL, AA, JM, VR, and BR conducted the experiments. SVP designed and directed the project. BMK and SVP wrote the manuscript with input from all the authors. All authors read and approved the final manuscript.

\section{ACKNOWLEDGMENTS}

We are thankful to the Whitehall Foundation, NIH (1R01MH094607-01A1 and 1R21MH096258-01A1) and The Scripps Research Institute for funding support, which enables us to carry out this research.

\section{SUPPLEMENTARY MATERIAL}

The Supplementary Material for this article can be found online at: http://www.frontiersin.org/journal/10.3389/fncel.2015. 00063/abstract

\section{DATA AVAILABILITY}

RNAseq data reported here is deposited in the GEO database with the accession number GSE61401.

\section{REFERENCES}

Anders, S., and Huber, W. (2010). Differential expression analysis for sequence count data. Genome Biol. 11:R106. doi: 10.1186/gb-2010-11-10-r106

Anders, S., Pyl, P. T., and Huber, W. (2015). HTSeq - a python framework to work with high-throughput sequencing data. Bioinformatics 31, 166-169. doi: 10.1093/bioinformatics/btu638

Cabili, M. N., Trapnell, C., Goff, L., Koziol, M., Tazon-Vega, B., Regev, A., et al. (2011). Integrative annotation of human large intergenic noncoding RNAs reveals global properties and specific subclasses. Genes Dev. 25, 1915-1927. doi: $10.1101 / \mathrm{gad} .17446611$

Cao, X., Yeo, G., Muotri, A. R., Kuwabara, T., and Gage, F. H. (2006). Noncoding RNAs in the mammalian central nervous system. Annu. Rev. Neurosci. 29, 77-103. doi: 10.1146/annurev.neuro.29.051605.112839

Carninci, P., Kasukawa, T., Katayama, S., Gough, J., Frith, M. C., Maeda, N., et al. (2005). The transcriptional landscape of the mammalian genome. Science 309, 1559-1563. doi: 10.1126/science.1112014

Clement-Ziza, M., Gentien, D., Lyonnet, S., Thiery, J. P., Besmond, C., and Decraene, C. (2009). Evaluation of methods for amplification of picogram amounts of total RNA for whole genome expression profiling. BMC Genomics 10:246. doi: 10.1186/1471-2164-10-246

Derrien, T., Johnson, R., Bussotti, G., Tanzer, A., Djebali, S., Tilgner, H., et al. (2012). The GENCODE v7 catalog of human long noncoding RNAs: analysis of their gene structure, evolution, and expression. Genome Res. 22, 1775-1789. doi: $10.1101 /$ gr.132159.111

Dieci, G., Fiorino, G., Castelnuovo, M., Teichmann, M., and Pagano, A. (2007). The expanding RNA polymerase III transcriptome. Trends Genet. 23, 614-622. doi: 10.1016/j.tig.2007.09.001
Faghihi, M. A., Modarresi, F., Khalil, A. M., Wood, D. E., Sahagan, B. G., Morgan, T. E., et al. (2008). Expression of a noncoding RNA is elevated in Alzheimer's disease and drives rapid feed-forward regulation of beta-secretase. Nat. Med. 14, 723-730. doi: 10.1038/nm1784

Guttman, M., Amit, I., Garber, M., French, C., Lin, M. F., Feldser, D., et al. (2009). Chromatin signature reveals over a thousand highly conserved large non-coding RNAs in mammals. Nature 458, 223-227. doi: 10.1038/nature07672

Halley, P., Kadakkuzha, B. M., Faghihi, M. A., Magistri, M., Zeier, Z., Khorkova, O., et al. (2014). Regulation of the apolipoprotein gene cluster by a long noncoding RNA. Cell Rep. 6, 222-230. doi: 10.1016/j.celrep.2013.12.015

Jeck, W. R., Sorrentino, J. A., Wang, K., Slevin, M. K., Burd, C. E., Liu, J., et al. (2013). Circular RNAs are abundant, conserved, and associated with ALU repeats. RNA 19, 141-157. doi: 10.1261/rna.035667.112

Jiang, X., Sunkara, N., Lu, S., and Liu, F. (2014). Directing RNase P-mediated cleavage of target mRNAs by engineered external guide sequences in cultured cells. Methods Mol. Biol. 1103, 45-56. doi: 10.1007/978-1-62703-730-3_4

Johnson, R. (2012). Long non-coding RNAs in Huntington's disease neurodegeneration. Neurobiol. Dis. 46, 245-254. doi: 10.1016/j.nbd.2011.12.006

Kadakkuzha, B. M., Akhmedov, K., Capo, T. R., Carvalloza, A. C., Fallahi, M., and Puthanveettil, S. V. (2013). Age-associated bidirectional modulation of gene expression in single identified R15 neuron of Aplysia. BMC Genomics 14:880. doi: 10.1186/1471-2164-14-880

Kadakkuzha, B. M., Liu, X. A., Narvaez, M., Kaye, A., Akhmedov, K., and Puthanveettil, S. V. (2014). Asymmetric localization of natural antisense RNA of neuropeptide sensorin in Aplysia sensory neurons during aging and activity. Front. Genet. 5:84. doi: 10.3389/fgene.2014.00084

Kapranov, P., Cheng, J., Dike, S., Nix, D. A., Duttagupta, R., Willingham, A. T., et al. (2007). RNA maps reveal new RNA classes and a possible function for pervasive transcription. Science 316, 1484-1488. doi: 10.1126/science.1138341

Kaushik, K., Leonard, V. E., Kv, S., Lalwani, M. K., Jalali, S., Patowary, A., et al. (2013). Dynamic expression of long non-coding RNAs (lncRNAs) in adult zebrafish. PLoS ONE 8:e83616. doi: 10.1371/journal.pone.0083616

Khaitovich, P., Kelso, J., Franz, H., Visagie, J., Giger, T., Joerchel, S., et al. (2006). Functionality of intergenic transcription: an evolutionary comparison. PLoS Genet. 2:e171. doi: 10.1371/journal.pgen.0020171

Khalil, A. M., Guttman, M., Huarte, M., Garber, M., Raj, A., Rivea Morales, D., et al. (2009). Many human large intergenic noncoding RNAs associate with chromatin-modifying complexes and affect gene expression. Proc. Natl. Acad. Sci. U.S.A. 106, 11667-11672. doi: 10.1073/pnas.0904715106

Kim, D., Pertea, G., Trapnell, C., Pimentel, H., Kelley, R., and Salzberg, S. L. (2013). TopHat2: accurate alignment of transcriptomes in the presence of insertions, deletions and gene fusions. Genome Biol. 14:R36. doi: 10.1186/gb-2013-144-r36

Kornienko, A. E., Guenzl, P. M., Barlow, D. P., and Pauler, F. M. (2013). Gene regulation by the act of long non-coding RNA transcription. BMC Biol. 11:59. doi: 10.1186/1741-7007-11-59

Kurokawa, R. (2011). Long noncoding RNA as a regulator for transcription. Prog. Mol. Subcell. Biol. 51, 29-41. doi: 10.1007/978-3-642-16502-3_2

Kutter, C., Watt, S., Stefflova, K., Wilson, M. D., Goncalves, A., Ponting, C. P., et al. (2012). Rapid turnover of long noncoding RNAs and the evolution of gene expression. PLoS Genet. 8:e1002841. doi: 10.1371/journal.pgen. 1002841

Li, L., Eichten, S. R., Shimizu, R., Petsch, K., Yeh, C. T., Wu, W., et al. (2014). Genome-wide discovery and characterization of maize long non-coding RNAs. Genome Biol. 15:R40. doi: 10.1186/gb-2014-15-2-r40

Livak, K. J., and Schmittgen, T. D. (2001). Analysis of relative gene expression data using real-time quantitative PCR and the 2(-Delta Delta C(T)) Method. Methods 25, 402-408. doi: 10.1006/meth.2001.1262

Lv, J., Cui, W., Liu, H., He, H., Xiu, Y., Guo, J., et al. (2013). Identification and characterization of long non-coding RNAs related to mouse embryonic brain development from available transcriptomic data. PLOS ONE 8:e71152. doi: 10.1371/journal.pone.0071152

Ma, L., Bajic, V. B., and Zhang, Z. (2013). On the classification of long non-coding RNAs. RNA Biol. 10, 925-933. doi: 10.4161/rna.24604

Mercer, T. R., Dinger, M. E., and Mattick, J. S. (2009). Long non-coding RNAs: insights into functions. Nat. Rev. Genet. 10, 155-159. doi: 10.1038/nrg2521

Mercer, T. R., Dinger, M. E., Sunkin, S. M., Mehler, M. F., and Mattick, J. S. (2008). Specific expression of long noncoding RNAs in the mouse brain. Proc. Natl. Acad. Sci. U.S.A. 105, 716-721. doi: 10.1073/pnas.0706729105 
Nakagawa, S., and Kageyama, Y. (2014). Nuclear IncRNAs as epigenetic regulators-Beyond skepticism. Biochim. Biophys. Acta 1839, 215-222. doi: 10.1016/J.Bbagrm.2013.10.009

Oka, T., Akisada, M., Okabe, A., Sakurai, K., Shiosaka, S., and Kato, K. (2002). Extracellular serine protease neuropsin (KLK8) modulates neurite outgrowth and fasciculation of mouse hippocampal neurons in culture. Neurosci. Lett. 321, 141-144. doi: 10.1016/S0304-3940(01)02470-3

Pauli, A., Valen, E., Lin, M. F., Garber, M., Vastenhouw, N. L., Levin, J. Z., et al. (2012). Systematic identification of long noncoding RNAs expressed during zebrafish embryogenesis. Genome Res. 22, 577-591. doi: 10.1101/gr. 133009.111

Ponjavic, J., Oliver, P. L., Lunter, G., and Ponting, C. P. (2009). Genomic and transcriptional co-localization of protein-coding and long non-coding RNA pairs in the developing brain. PLoS Genet. 5:e1000617. doi: 10.1371/journal.pgen.1000617

Prasanth, K. V., Prasanth, S. G., Xuan, Z., Hearn, S., Freier, S. M., Bennett, C. F., et al. (2005). Regulating gene expression through RNA nuclear retention. Cell 123, 249-263. doi: 10.1016/j.cell.2005.08.033

Puthanveettil, S. V., Antonov, I., Kalachikov, S., Rajasethupathy, P., Choi, Y. B., Kohn, A. B., et al. (2013). A strategy to capture and characterize the synaptic transcriptome. Proc. Natl. Acad. Sci. U.S.A. 110, 7464-7469. doi: 10.1073/pnas. 1304422110

Qureshi, I. A., Mattick, J. S., and Mehler, M. F. (2010). Long non-coding RNAs in nervous system function and disease. Brain Res. 1338, 20-35. doi: 10.1016/j.brainres.2010.03.110

Ren, A., Zhang, H., Xie, Z., Ma, X., Ji, W., He, D. Z., et al. (2012). Regulation of hippocampus-dependent memory by the zinc finger protein Zbtb20 in mature CA1 neurons. J. Physiol. 590, 4917-4932. doi: 10.1113/jphysiol.2012. 234187

Roberts, T. C., Morris, K. V., and Wood, M. J. (2014). The role of long non-coding RNAs in neurodevelopment, brain function and neurological disease. Philos. Trans. R. Soc. Lond. B. Biol. Sci. 369. doi: 10.1098/rstb.2013.0507

Robinson, J. T., Thorvaldsdottir, H., Winckler, W., Guttman, M., Lander, E. S., Getz, G., et al. (2011). Integrative genomics viewer. Nat. Biotechnol. 29, 24-26. doi: $10.1038 /$ nbt. 1754

Sauvageau, M., Goff, L. A., Lodato, S., Bonev, B., Groff, A. F., Gerhardinger, C., et al. (2013). Multiple knockout mouse models reveal lincRNAs are required for life and brain development. Elife 2:e01749. doi: 10.7554/eLife.01749

Soreq, L., Guffanti, A., Salomonis, N., Simchovitz, A., Israel, Z., Bergman, H., et al. (2014). Long non-coding RNA and alternative splicing modulations in Parkinson's leukocytes identified by RNA sequencing. PLoS Comput. Biol. 10:e1003517. doi: 10.1371/journal.pcbi.1003517

Trapnell, C., Williams, B. A., Pertea, G., Mortazavi, A., Kwan, G., Van Baren, M. J., et al. (2010). Transcript assembly and quantification by RNA-Seq reveals unannotated transcripts and isoform switching during cell differentiation. Nat. Biotechnol. 28, 511-515. doi: 10.1038/nbt.1621

Van Heesch, S., Van Iterson, M., Jacobi, J., Boymans, S., Essers, P. B., De Bruijn, E., et al. (2014). Extensive localization of long noncoding RNAs to the cytosol and mono- and polyribosomal complexes. Genome Biol. 15:R6. doi: 10.1186/gb2014-15-1-r6

Wang, K. C., and Chang, H. Y. (2011). Molecular mechanisms of long noncoding RNAs. Mol. Cell 43, 904-914. doi: 10.1016/j.molcel.2011.08.018

Wang, M. E., Fraize, N. P., Yin, L., Yuan, R. K., Petsagourakis, D., Wann, E. G., et al. (2013). Differential roles of the dorsal and ventral hippocampus in predator odor contextual fear conditioning. Hippocampus 23, 451-466. doi: 10.1002/hipo. 22105

Wang, S., Wang, L., Zhu, T., Gao, X., Li, J., Wu, Y., et al. (2010). Improvement of tissue preparation for laser capture microdissection: application for cell type-specific miRNA expression profiling in colorectal tumors. BMC Genomics 11:163. doi: 10.1186/1471-2164-11-163

Wu, L., Murat, P., Matak-Vinkovic, D., Murrell, A., and Balasubramanian, S. (2013). Binding interactions between long noncoding RNA HOTAIR and PRC2 proteins. Biochemistry 52, 9519-9527. doi: 10.1021/bi4 $01085 \mathrm{~h}$

Zhang, B., Arun, G., Mao, Y. S., Lazar, Z., Hung, G., Bhattacharjee, G., et al. (2012). The lncRNA Malatl is dispensable for mouse development but its transcription plays a cis-regulatory role in the adult. Cell Rep. 2, 111-123. doi: 10.1016/j.celrep.2012.06.003

Conflict of Interest Statement: The authors declare that the research was conducted in the absence of any commercial or financial relationships that could be construed as a potential conflict of interest.

Received: 17 December 2014; accepted: 10 February 2015; published online: 06 March 2015.

Citation: Kadakkuzha BM, Liu X-A, McCrate J, Shankar G, Rizzo V, Afinogenova A, Young B, Fallahi M, Carvalloza AC, Raveendra B and Puthanveettil SV (2015) Transcriptome analyses of adult mouse brain reveal enrichment of lncRNAs in specific brain regions and neuronal populations. Front. Cell. Neurosci. 9:63. doi: 10.3389/fncel. 2015.00063

This article was submitted to the journal Frontiers in Cellular Neuroscience. Copyright (c) 2015 Kadakkuzha, Liu, McCrate, Shankar, Rizzo, Afinogenova, Young, Fallahi, Carvalloza, Raveendra and Puthanveettil. This is an open-access article distributed under the terms of the Creative Commons Attribution License (CC BY). The use, distribution or reproduction in other forums is permitted, provided the original author(s) or licensor are credited and that the original publication in this journal is cited, in accordance with accepted academic practice. No use, distribution or reproduction is permitted which does not comply with these terms. 